\title{
DOSSIER
}

\section{TIEMPOS DE OCUPACIÓN: EXPERIENCIAS, ROSTROS Y ESPACIOS EN UNA EUROPA EN GUERRA (1936-1945) INTRODUCCIÓN}

\section{Times of Occupation: Experiences, Faces and Spaces in Europe at War}

(1936-1945)

An Introduction

Coordinado por

\section{Alejandro Pérez-Olivares}

(Sciences Po Lyon)

\section{PRESENTACIÓN}

\section{DOI: https://doi.org/10.20318/hn.2021.5890}

\begin{abstract}
Resumen: Este texto presenta los artículos que componen el dossier "Tiempos de ocupación: experiencias, rostros y espacios en una Europa en guerra (1936-1945)". En este sentido, se centra en las principales problemáticas que permiten comprender las ocupaciones en el marco de la "guerra total". Este fenómeno transnacional es abordado través de diferentes estudios de caso en España, Países Bajos y Francia.
\end{abstract}

Palabras clave: Ocupaciones - Guerra total "Guerra civil europea" - Historia transnacional.
Abstract: This text introduces the contributions that make up this special issue called 'Times of occupation: Experiences, Faces and Spaces in Europe at war (1936-1945)'. In this sense, it focuses on the main questions that allow us to understand the occupations during the 'total war era', a transnational reality which is addressed thanks to different case studies in Spain, the Netherlands and France.

Keywords: Occupations - Total War - "European Civil War" - Transnational History. 
“...supone mucho tiempo y a veces duele el hombro al estirar el cuello; pero así se descubre un sentido”.

(Arlette Farge, La atracción del archivo) ${ }^{1}$.

“A causa del muro y la falta de permisos, mi hija Amna' dejó el colegio cuando tenía 15 años”. Hace diez años, Um Ibrahim tenía claro cuál de las expresiones de la ocupación había afectado más a su vida cotidiana y la de su familia. "Es más probable que las niñas pierdan clase y abandonen porque, a diferencia de los niños, son menos propensos a escalar el muro". En la ciudad de Bir Nabala, el paseo de diez minutos hasta la escuela se vio transformado en un viaje de una hora o incluso más, en función del tiempo de espera en el puesto de control, salvo que se optara por la opción de saltar el muro, ahorrando bastantes minutos y burlando la vigilancia. La restricción en el acceso a la educación es sólo uno de los elementos que definen la vida en los territorios ocupados por Israel desde 1967: el día a día en Jerusalén Este también se define por el control del espacio a través de los asentamientos y las barreras o de la propia población a través de la revocación de sus documentos de identidad, dificultando la movilidad ${ }^{2}$. La realidad cotidiana de la ocupación de los territorios palestinos revela un proceso histórico mayor, el colonialismo de asentamiento, que hunde sus raíces a finales del siglo XIX y que explica en gran parte la masiva expulsión de población local desde mediados del siglo XX, la constante adaptación a nuevas realidades o la relevancia del paisaje en la siempre conflictiva gestión de la memoria con las autoridades de ocupación. Un paisaje que no es interpretado o experimentado de la misma manera por militares y civiles, aunque compartan los mismos lugares, y donde planos, líneas y puntos se suman al tiempo para construir una cartografía particular ${ }^{3}$.

La compleja relación entre las autoridades militares y civiles también se proyecta sobre otra de las ocupaciones más adheridas al imaginario colectivo reciente.

\footnotetext{
${ }^{1}$ Arlette Farge, La atracción del archivo (Valencia, Edicions Alfons el Magnànim, 1991), 18.

2 Oficina de Coordinación de Asuntos Humanitarios, East Jerusalem. Key Humanitarian Concerns (ONU, 2011). La historia de Um Ibrahim y sus hijas en pp. 94-95.

${ }^{3}$ Jorge Ramos Tolosa, "Propuestas para decolonizar Palestina-Israel”, en Epistemologías del Sur, eds. Maria Paula Meneses y Karina Bidaseca (Buenos Aires : Coimbra, CLACSO, 2018); Shelley Egoz y Tim Williams, "Coexistent Landscapes: Military Integration and Civilian Fragmentation", en Militarized Landscapes. From Gettysburg to Salisbury Plan, Ed. Chris Pearson, Peter Coates y Tim Cole (London : New York, Continuum Books, 2010).
} 
La guerra de Irak de 2003 se ha comprendido principalmente a partir de la ideología de los poderes ocupantes, lo que explica el deseo de comenzar una maniobra de ocupación (la teoría de la "guerra preventiva"), y de los intereses concretos a la hora de llevarla a cabo (la gestión directa de fuentes petrolíferas). Sin embargo, ambas realidades no pueden desligarse de un contexto geopolítico específico que las dota de contenido y explica no sólo la forma en que la ocupación fue llevada a cabo, sino también la larga y dificultosa reconstrucción de las instituciones iraquíes después. En ese sentido, no siempre es sencillo de definir el traspaso de competencias desde la esfera militar a la civil. Tampoco de ejecutar en la práctica. Cuestiones como asegurar la gobernabilidad de un territorio y una sociedad, constituir una nueva institucionalidad, afirmar su funcionamiento cotidiano y su relación con la población, gestionar la seguridad después de un conflicto o administrar la ayuda humanitaria hacen que una etapa y otra estén separadas por un mero "traspaso de poderes" 4 . Todo ello hace que la relación entre los poderes civiles locales y las autoridades de ocupación pueda ser interpretada como la cesión de los primeros ante la imposición de los segundos ${ }^{5}$.

Las ocupaciones, de este modo, conectan diferentes realidades en una sociedad a partir del despliegue del Ejército, una práctica tradicionalmente asociada a un contexto bélico pero que puede ser también ejercida en tiempo de paz. En este segundo caso, los regímenes liberales recurren a mecanismos legales que sancionan diferentes formas de estado de excepción, como es el caso de Francia desde 2015 debido a la amenaza terrorista ${ }^{6}$. Otros ejemplos recientes muestran la complicada puesta en práctica de este tipo de decisiones. El paso del huracán Katrina por Nueva Orleans obligó al desplazamiento forzado de un millón de personas y generó una falta de agua potable y

\footnotetext{
${ }^{4}$ Raymond Hinnebusch, "The US Invasion of Iraq: Explanations and Implications", Critique: Critical Middle Eastern Studies (2007), 209-228; Joseph J. Collins, Choosing War. The Decision to Invade Iraq and its Aftermath (Washington DC, National Defense University Press, 2008), pp. 11-16.

${ }^{5}$ Así fue cómo se expresó Sheikh Mohannad Al-Gazawi, uno de los miembros del Movimiento Sadrista, el partido político iraquí de la mayoría chií, liderado por el clérigo Muqtada al-Sadr: el acuerdo con Estados Unidos sobre el mantenimiento de las tropas tras la invasión era "un desastroso acuerdo que destroza Irak y cede ante la potencia ocupante". Puede verse "Iraqis Protest Against US Military Deal", Global Policy Forum, https://www.globalpolicy.org/component/content/article/168/37378.html, 30 de mayo de 2008.

${ }^{6}$ En este caso, l'état d'urgence fue creado como instrumento en 1955, durante la guerra de Argelia (19541962), y su relevancia política entre la IV y la V República sigue siendo objeto de debate. Puede verse al respecto Sylvie Thénault, "L'état d'urgence (1955-2005). De l'Algérie coloniale à la France contemporaine: destin d'une loi", Le Mouvement Social, 218/1 (2007), 63-78.
} 
electricidad generalizada. El alcalde de la ciudad y el gobernador de Louisiana recurrieron a la ayuda del gobierno federal, que decidió desplegar a la Guardia Nacional (inicialmente, más de 5000 soldados, llegando a la cifra de 41000 apenas una semana más tarde) y coordinar las acciones desde el Departamento de Seguridad Nacional. Sin embargo, la actuación conjunta del Ejército y las fuerzas de policía adoleció de una falta de liderazgo y de capacidad de controlar las realidades cotidianas de la tragedia: en la ciudad, la policía local no podía comunicarse con el resto de agencias de control desplegadas en el área (algunas de ellas, privadas), lo que provocó la duplicación de esfuerzos y la interferencia en las competencias particulares ${ }^{7}$. La suspensión de ciertos derechos de ciudadanía se concretó en la diferente capacidad de movilidad a lo largo de la ciudad, la segregación en el trato en los puestos de control, el escaso (y tardío) control de la actuación policial y la redefinición de la autoridad en Nueva Orleans, lo que para algunos convirtió la ciudad en una zona de guerra ${ }^{8}$.

En este sentido, el "estado de excepción" no se refiere únicamente a la súbita alteración de las "viejas" instituciones o la constitución de otras "nuevas" en un tiempo breve, sino sobre todo a la penetración de las nuevas normas en la vida cotidiana, a la posibilidad de adaptarse a las mismas o al surgimiento de formas originales de resistencia, colaboración o elusión, o a la identificación de nuevos conflictos, nuevos debates y nuevas oportunidades políticas. La actual crisis sanitaria de la COVID-19 ha revelado asimismo formas peculiares de expresar la autoridad y nuevas proyecciones de las relaciones de poder sobre el espacio y el tiempo ${ }^{9}$. Prácticas recientes como el

\footnotetext{
${ }^{7}$ Christopher Oscar, Post Katrina Redefining the Military Role in Homeland Security (US Army War College, Carlisle, 2006); Steve Bowman, Lawrence Kapp y Amy Belasco, Hurricane Katrina: DOD Disaster Response (Washington, The Library of Congress, 2005), https://fas.org/sgp/crs/natsec/RL33095.pdf.

8 "New Orleans uses martial law to deal with chaotic situation", Los Angeles Times, 31 de Agosto de 2005; "Military occupation turns New Orleans into war zone", Financial Times, 6 de septiembre de 2005. Este sentido restrictivo entronca con la interpretación que Agamben hace del "estado de excepción", entendido como la suspensión de un orden jurídico en la práctica y comprendido a partir de la interrelación de las instituciones, las normas y las prácticas sociales. Puede verse Giorgio Agamben, Estado de excepción. Homo Sacer II, 1(Madrid, Pre-Textos, 2010).

${ }^{9}$ Giorgio Agamben, "Reflexiones sobre la peste", en Sopa de Wuhan, Giorgio Agamben et al. (Editorial ASPO, 2020), 135-137; Ivan Franch-Pardo et al., "Spatial Analysis and GIS in the study of COVID-19. A study", Science of the Total Environment, 739 (2020), doi: https://doi.org/10.1016/j.scitotenv.2020.140033. Sobre los abusos de autoridad en este contexto puede verse Amnistía Internacional, Violaciones de derechos humanos durante el estado de alarma (Madrid, 2020),

https://www.es.amnesty.org/fileadmin/user upload/Violaciones ddhh estado de alarma junio 2020.pdf
} 
confinamiento o el mantenimiento de la "distancia de seguridad", la orientación del itinerario en centros educativos o la fijación de lugares de espera en estaciones de tren, metro y tranvía, suponen una actualización de los significados asociados no hace tanto tiempo a las categorías de espacio concebido, espacio percibido y espacio vivido. La nueva concepción de nuestros lugares cotidianos, condicionada por la pandemia, también está relacionada con una nueva consideración del tiempo, de su importancia social y su gestión en términos afectivos. Entendido en términos sociales, el tiempo alude a las posibilidades para la movilidad, a las formas de sociabilidad y consumo, a las prácticas que están reconocidas y gozan de un consenso mayoritario o a la propia ordenación del territorio, que facilita o dificulta según qué desplazamientos ${ }^{10}$.

$* * * * *$

Es probable pensar que las ocupaciones son realidades del pasado. Los cuatro ejemplos anteriores, junto a muchos otros más, demuestran sin embargo que sólo hace falta "estirar el cuello" y mirar alrededor para descubrir que no es así. Este dossier está atravesado por cuestiones como la "densidad" histórica que revelan las ocupaciones y sus efectos en las relaciones sociales o en las actitudes cotidianas, como demuestra el caso de Palestina, o el encuadramiento de cualquier ocupación en un marco geopolítico mayor, como se deduce de la experiencia de la última guerra de Irak. También por los conflictos derivados de la reconsideración del concepto de autoridad, del choque entre “nuevas" y "viejas autoridades" y su difícil acoplamiento, y por la proyección de las relaciones de poder en la gestión del espacio y del tiempo, como muestran contextos tan particulares como el desastre natural que asoló Nueva Orleans hace tres lustros o la actual pandemia global. Las diferentes contribuciones de este dossier expresan estas preocupaciones enfocando a un tiempo específico: los últimos compases de la "guerra total” en Europa que, tal y como propuso Sophie De Schaepdrijver hace algunos años, estuvo protagonizada por diversas formas de ocupación. En este sentido, tanto en los

10 Antonio Scala et al., "Time, space and social interactions: exit mechanisms for the Covid-19 epidemics", Scientific Reports, 10 (2020), doi: https://doi.org/10.1038/s41598-020-70631-9; Paisaje Transversal, "La ciudad del Coronavirus", https://paisajetransversal.org/2020/07/la-ciudad-delcoronavirus/, 17 de julio de 2020. 
ciclos de "guerra total" de 1914-1918 y 1936-1945 como en otros de conflictos de baja intensidad o incluso en los periodos de paz, la ocupación se reveló como un fenómeno transversal a la primera mitad del siglo XX. Escenarios y tensiones muy diversos se saldaron igualmente con el despliegue de soldados en territorios ajenos en busca del "orden" y con una vida cotidiana marcada por la siempre difícil relación entre los militares y la población civil ${ }^{11}$.

De acuerdo con Dennis Showalter, la ocupación como concepto es tan antigua como la guerra misma, pero apenas "algo más joven que la idea de una victoria decisiva". Las ocupaciones suelen hacer referencia a la intención de dominar, desplazar o reemplazar a los habitantes de un territorio; a la sensación de seguridad o inseguridad en un sentido geopolítico; a la explotación de los recursos financieros, industriales o agrícolas o, según el origen ideológico de su justificación, también aluden al "progreso" o a la fundación de un imperio ${ }^{12}$. Esta mirada permite comparar diferentes experiencias pero, al mismo tiempo, reduce las ocupaciones a fenómenos asociados a la acción estatal y al despliegue de sus herramientas, normalmente en referencia a la actuación de los poderes ocupantes. En 1944, mientras los Aliados ultimaban la ocupación de Alemania durante los últimos compases de la II Guerra Mundial, el jurista y sociólogo Ernst Fraenkel reflexionó sobre la necesaria relación entre los términos "ocupación" e "imperio de la ley", una forma de interpretar este fenómeno a través del derecho y la ciencia política que permeó durante largo tiempo la historiografía en la segunda mitad de siglo ${ }^{13}$. Sin embargo, los debates historiográficos recientes han mostrado cómo las

\footnotetext{
${ }^{11}$ Sophie De Schaepdrijver, "Military occupations, 1914-1945", en The Cambridge History of War, Vol. $I V$. War and the Modern World, eds. Roger Chickering, Dennis Showalter y Hans Van De Ven (Cambridge, Cambridge University Press, 2012), 236-256 y "Populations under occupation", en The Cambridge History of the First World War. Vol. III: Civil Society, ed. Jay Winter (Cambridge, Cambridge University Press, 2014), 242-256.

${ }^{12}$ Dennis Showalter, "Military occupations, 1945-1955", en The Cambridge History of War, Vol. IV, 428451; Marcel Boldorf y Tetsuki Okazaki eds., Economies Under Occupation. The Hegemony of Nazi Germany and Imperial Japan in World War II (London: New York, Routledge, 2015); Mark Mazower, El imperio de Hitler (Barcelona, Crítica, 2008).

${ }^{13}$ Ernst Fraenkel, Military Occupation and the Rule of Law (New York, Oxford University Press, 1944); Odile Debrash, L'occupation militaire (Paris, Librairie générale de droit et de jurisprudence, 1962). En perspectiva, ambos trabajos eran deudores del contexto de entreguerras, pero también de la forma en que se explicaban las ocupaciones de finales del siglo XIX, en particular la que puso fin a la guerra franco-prusiana. Treinta años antes de la publicación del libro de Fraenkel, Robin Raymond analizó las ocupaciones únicamente a partir del derecho internacional. Un año después, la "Gran Guerra" alteró
} 
ocupaciones son un momento predilecto para la expresión de las diversas tensiones preexistentes en una sociedad, donde la población reacciona de manera diversa a los poderes ocupantes y donde el propio éxito de la ocupación depende, en gran medida, de esa relación. De hecho, no es casual que entre mediados de la década de 1930 y finales de la década de 1940, las ocupaciones fueran una manifestación específica de la "guerra civil europea", de las profundas divisiones que experimentó el continente en términos ideológicos, sociales, culturales y políticos. Por eso es preferible comprender estos contextos tan particulares desde la consideración amplia de los "regímenes de ocupación", es decir, el conjunto de instituciones, normas y propósitos implicados en la gestión de un territorio "nuevo", "ajeno" o "enemigo"14.

Los textos que ahora tengo el placer de introducir aluden a este sentido histórico, concreto, y componen una panorámica compleja de un fenómeno transnacional a partir de tres casos de estudio: España, Países Bajos y Francia. Ocho aportaciones enmarcadas entre el golpe de Estado de 1936 contra la II República española, por un lado, y el final del régimen de ocupación nazi en 1945, por otro. Entre una fecha y otra, la "guerra total" se expresó de diferentes formas, requirió diversas herramientas y legó múltiples consecuencias en los tres países; se constituyeron nuevas instituciones o se reaprovecharon otras antiguas para asentar tres regímenes de ocupación con tantas semejanzas como diferencias y se concitaron diferentes formas de colaboración, adaptación, resistencia o persecución entre quienes fueron invadidos y los nuevos gestores del orden. Entre 1936 y 1945, las realidades española, holandesa y francesa estuvieron atravesadas por múltiples formas de violencia que provocaron diferentes reacciones y divisiones de la sociedad ${ }^{15}$. Así, esta reflexión coral ofrece una

trágicamente este tipo de explicaciones. Puede verse Robin Raymond, Des occupations militaires en dehors des occupations de guerre (Paris, Larose \& Tenin, 1913).

${ }^{14}$ La definición de "regimen de ocupación" a partir de Cor Lammers, "Levels of Collaboration: A Comparative Study of German Occcupation Regimes during the Second World War", The Netherlands Journal of Social Sciences, 31 (1995), 3-31. Para la relación entre ocupaciones y guerras civiles, un balance reciente y sugerente es Javier Rodrigo y David Alegre, Comunidades rotas. Una historia global de las guerras civiles, 1917-2017 (Barcelona, Galaxia Gutenberg, 2019), 167-287. La última expresión entrecomillada y su desarrollo en el ya clásico Enzo Traverso, A sangre y fuego. De la guerra civil europea, 1914-1945 (Valencia, Publicacions de la Universitat de València, 2009).

15 Gutmaro Gómez Bravo y Jorge Marco, La obra del miedo. Violencia y sociedad en la España franquista, 1936-1950 (Barcelona, Península, 2011); Peter Romijn e Ismee Tames, "Transnational Identities of Dutch-Nazi Collaborators and their Struggle for Integration into the National Community", Journal of Modern European History, 16/2 (2018), 247-268; François Broche, Dictionnaire de la Collaboration. Collaborations, compromissions, contradictions (Paris, Belin, 2014). 
interpretación plural de uno de los fenómenos que marcaron la historia europea del siglo $\mathrm{XX}$, y lo hace siguiendo algunas de las recomendaciones sugeridas por el historiador francés Philippe Burrin: comprender las ocupaciones a través de la comparación, mostrar los "efectos estructurales" de las ocupaciones en el ambiente general de la sociedad y sus condiciones de vida y no olvidar las interacciones cotidianas entre ocupantes y ocupados ${ }^{16}$.

En este sentido, el principal objetivo de este número es reevaluar una serie de nociones tradicionalmente asentadas en las historiografias española, francesa $\mathrm{y}$ holandesa a la luz de un conjunto de realidades que definieron un proceso más amplio. En el caso español, hace unos años François Godicheau apuntó que las diferentes denominaciones que recibió el conflicto inaugurado por la sublevación militar de julio permiten hablar del "carácter inacabado" del concepto de "guerra civil". De este modo, la propia denominación con la que nos referimos al ciclo 1936-1939 "no funciona de manera aislada sino como elemento de una pareja fundamental de orden y desorden”, es decir, en referencia a los distintos proyectos políticos que pretendían orientar la sociedad en un sentido u otro, una realidad que recorrió las tensiones de la Europa del momento y se expresó abiertamente durante las ocupaciones ${ }^{17}$. La forma de definir "nuestra" guerra en aquel contexto compartido es todavía un proceso abierto, producto también del debate público y de la gestión de su legado traumático, pero como plantea Pablo Sánchez León la utilización acrítica del sintagma "guerra civil” corre el peligro de "oscurecer" la complejidad de todas sus dimensiones, de limitar "todas las guerras" que podríamos contar y comprender ${ }^{18}$. Entre ellas, destaca en los últimos años la

\footnotetext{
16 Philippe Burrin, "Writing the History of Military Occupations", en France at War: Vichy and the Historians, ed. Sarah Fishman (Oxford, Berg, 2000), 77-90. La cita entrecomillada en 80.

${ }^{17}$ François Godicheau, "La Guerra Civil, figura del desorden público. El concepto de guerra civil y la definición del orden político", en Guerras civiles. Una clave para entender la Europa de los siglos XIX y $X X$, eds. Jordi Canal y Eduardo González Calleja (Madrid, Casa de Velázquez, 2012), 75-88. Esta reflexión se enmarca en la estela de otros trabajos previos, como los de Rafael Cruz o del propio Godicheau. Véase Rafael Cruz, En el nombre del pueblo. República, rebelión y guerra en la España de 1936 (Madrid, Siglo XXI, 2006) y François Godicheau, "Guerra civil, guerra incivil: la pacificación por el nombre", en Guerra civil: mito y memoria, coord. François Godicheau y Julio Aróstegui (Madrid, Marcial Pons, 2006), 137-166.

${ }^{18}$ Entre otros motivos, porque los propios contendientes no utilizaron esa expresión durante el conflicto. Véase Pablo Sánchez León, "Erradicar la ciudadanía: 1936 más allá de una guerra civil”, en La guerra que nos han contado y la que no. Memoria e historia de 1936 para el siglo XXI, Pablo Sánchez León y Jesús Izquierdo Martín (Madrid, Postmetrópolis, 2017), 305-380. Sobre las diferentes denominaciones de
} 
consideración y conceptualización de la Guerra Civil española como una clara guerra de ocupación, una realidad que se proyectó tanto a las diversas lógicas de la violencia como a la imposición del orden en los territorios conquistados, lo que ha difuminado las fronteras que separan la guerra y la posguerra y ha permitido hablar de la existencia de una "guerra civil irregular" hasta la década de 1950 .

La reflexión acerca de la experiencia española se abre con la aportación de Guillermo Pastor, Henar Alonso y Carmen Rial sobre la forma en que los archivos revelan la naturaleza del orden social que los genera, una realidad muy pocas veces atendida y sin embargo crucial para entender tanto el propio trabajo historiográfico como la gestión del patrimonio documental. La doble consideración objeto-sujeto del archivo se presenta a través de tres ejemplos que permiten reinterpretar la Guerra Civil como una "guerra total" y de ocupación. La secuencia negación - construcción - exilio de archivos que los autores desarrollan en primer lugar alude a las profundas divisiones que recorrieron la sociedad española en aquellos años (y después), de modo que la inestabilidad de la retaguardia republicana se proyectó también sobre la genealogía de la documentación generada por ella. En segundo lugar, la necesidad de información para perseguir al "enemigo" y la forma predominante que adoptó su castigo definieron las sucesivas ocupaciones de territorios por parte del Ejército sublevado, y marcaron el conflicto también en un sentido archivístico. Así, el monopolio de los servicios de inteligencia por parte del Servicio de Información y Policía Militar (SIPM) y el despliegue de la maquinaria punitiva franquista a través de los consejos de guerra, dos de los fondos documentales más novedosos y abiertos a la consulta más recientemente, completan esta aportación. Un trabajo que entiende la ocupación como un momento claramente "constituyente", de creación de nuevas instituciones y coordinación de diferentes políticas de control social, y que deja una decidida impronta documental.

la Guerra Civil española (y los contextos de creación y difusión de los mismos), es imprescindible Javier Rodrigo, Cruzada, paz, memoria. La Guerra Civil en sus relatos (Granada, Comares, 2013).

19 Jorge Marco, "Rethinking the Postwar Period in Spain: Violence and Irregular Civil War, 1939-52", Journal of Contemporary History, 55/3 (2019), 492-513; Gutmaro Gómez Bravo, Geografía humana de la represión franquista. Del golpe a la guerra de ocupación, 1936-1941 (Madrid, Cátedra, 2017); Miguel Alonso Ibarra, "Combatir, ocupar, fusilar. La evolución de la violencia bélica de los sublevados en la guerra civil española (1936-1939)", en Europa desgarrada: guerra, ocupación y violencia (1900-1950), coord. David Alegre Lorenz, Miguel Alonso Ibarra y Javier Rodrigo (Zaragoza, Prensas Universitarias de Zaragoza, 2018); Alejandro Pérez-Olivares, Madrid cautivo. Ocupación y control de una ciudad, 1936-1948 (Valencia, Publicacions de la Universitat de València, 2020). 
El artículo de Carlos Píriz también se interroga por el relevante papel de la inteligencia militar en el marco de la "guerra total". En este caso, el autor enfoca esta cuestión general a partir de un contexto muy específico, el de la entrada del ejército franquista en Madrid al final de la Guerra Civil, y desde el cual pone en valor el contacto con la retaguardia "enemiga" como un fenómeno esencial para comprender la preparación, el despliegue y el éxito de la ocupación. Este trabajo presenta la noción de (pre)ocupación a modo de "descripción densa" para analizar la relevancia de la primera gestión y el control de realidades clave en el mundo urbano, como los medios de comunicación, el abastecimiento o el transporte. Una vez más, el concurso del Servicio de Información y Policía Militar (SIPM) fue clave para entender este proceso, así como su conexión orgánica con la famosa "Quinta Columna", de la que el autor es un reconocido especialista. Por último, este texto hace de las ocupaciones durante la Guerra Civil española una oportunidad para el intercambio de experiencias y saberes clave en el desarrollo de la guerra moderna, y para ello lo entiende desde la conformación de una "red" al tiempo que abre el contexto concreto de marzo de 1939 a la posibilidad de la comparación internacional.

Si el texto anterior amplía la escala de análisis para reducir el objeto de estudio, la contribución de Alba Fernández sitúa la ocupación de las instituciones académicas en perspectiva a partir de su investigación sobre la producción historiográfica en el Consejo Superior de Investigaciones Científicas. Así, el CSIC sirve de ejemplo para reflejar la necesidad que el "nuevo Estado" franquista tuvo de crear una institucionalidad propia, marcada tanto por las rupturas como por las continuidades con las décadas anteriores. Según la autora, la creación del Consejo sólo unos meses después de finalizar la Guerra Civil puede interpretarse como un episodio más en la trayectoria del nacionalcatolicismo académico, que al fin controlaba los espacios de producción del conocimiento. Sin embargo, este artículo permite dibujar un escenario aún más complejo para el análisis de las instituciones culturales de la dictadura y la ponderación de las trayectorias de gran parte de sus miembros. De este modo, mientras que el discurso público franquista sostenía la ruptura con las instituciones, los modos de hacer y las personalidades que habían protagonizado el despegue científico español en el primer tercio del siglo XX, tanto la necesidad de poner en marcha los patronatos e institutos del CSIC como la propia práctica historiográfica de posguerra dibujaron más 
líneas de continuidad de las que a priori podría pensarse. Puede que el pasado liberal les resultara incómodo a las autoridades del régimen pero, en palabras de la propia autora, la "aculturación por oposición al periodo anterior" convivió con otras realidades más funcionales que también definieron la constitución del orden académico franquista.

La difícil imposición del orden entre 1940 y 1944 fue una de las características del régimen de ocupación nazi en Francia. Como ya adelantó hace una década Kenneth Mouré para el caso de París, esta inestabilidad, extrapolable al conjunto del país, puede explicarse a través de cuatro grandes factores: la penuria económica que acompañó a la ocupación, principalmente a partir del ecuador de la II Guerra Mundial; la compleja estructura del poder vigilante y la debilidad del régimen de Vichy, a medio camino entre las autoridades locales y foráneas, o lo que cada una de ellas entendió como actitudes legales o ilegales, clasificadas como delito ${ }^{20}$. De creer los testimonios que nos han legado esos cuatro años de ocupación, hubo tantas rupturas como continuidades, tanta "normalidad" como conmoción. La recuperación de las actitudes cotidianas, aquellas "vidas gobernadas por circunstancias más allá de su control”, permite trascender el binomio ocupación - resistencia para enfocar a "las limitaciones y los problemas a los que se enfrentó cada individuo". Quizá uno de los ejemplos más representativos de este enfoque sea el trabajo de Robert Gildea, uno de los primeros autores en plantearse la relevancia de los diferentes espacios compartidos por ocupantes y ocupados, de modo que la "cohabitación" puede ser considerada como una imagen certera de la complejidad de la experiencia de ocupación ${ }^{21}$. Apostar por esta perspectiva supone, en suma, desmarcarse tanto de las imágenes oscuras de 1940 como de las alegres de 1944 y afirmar que, a diferencia del marco interpretativo emanado del "mito gaullista" en la

\footnotetext{
${ }^{20}$ Kenneth Mouré, "The Faux Policier in Occupied Paris", Journal of Contemporary History, 45/I (2010), 95-112. El estudio de Kitson sobre la policía de París y la cuantificación que aportó Laub abonan esta tesis. Puede verse Simon Kitson, "The Police in the Liberation of Paris", en The Liberation of France. Image and Event, ed. H. R. Kedward y Nancy Wood (Oxford : Herndon, Berg, 1995), 43-56 y Thomas J. Laub, After the Fall. German Policy in Occupied France, 1940-1944 (New York, Oxford University Press, 2010), especialmente 104-110.

${ }^{21}$ Robert Gildea, Marianne in Chains. Daily Life in the Heart of France During the German Ocupation (London, MacMilan, 2002). Los entrecomillados previos en Richard Vinen, The Unfree French. Life Under Occupation (London, Penguin, 2007), 2.
} 
segunda posguerra mundial, tanto en una fecha como en otra Francia fue ocupada militarmente ${ }^{22}$.

Dos de las contribuciones de este dossier profundizan en algunas de estas problemáticas. Jean Marc Berlière se ocupa de las transformaciones experimentadas por la Policía francesa, bien como institución estatal a medio camino entre el régimen de ocupación nazi y el de Vichy, bien como colectivo social a partir de preguntarse por las motivaciones de los propios agentes para ejecutar las políticas represivas y excluyentes de las respectivas autoridades. Desde esta perspectiva, el estudio de la Policía permite ensanchar la brecha analítica entre la colaboración y la resistencia como actitudes hegemónicas durante la ocupación y trascender el mero estudio de discursos y disposiciones para plantear los conflictos que generó este contexto: las diversas funciones atribuidas, la desconfianza hacia los nuevos responsables de la gestión del orden, la privatización de la función policial (las "policías de ocasión") o la forma específica que adoptaron procesos de mayor recorrido como la centralización de la institución o la modernización de sus repertorios de acción. Este trabajo, por tanto, superpone dos planos analíticos: uno más coyuntural, interesado en el tiempo corto de la ocupación; otro más estructural, volcado en la evaluación de su impacto en la trayectoria particular de la Policía. La combinación de ambos acercamientos permite desmentir tanto la tesis de la colaboración masiva de esta institución con los ocupantes como el mito construido después de la "Liberación" de 1944, que situó a los agentes de policía en las filas de la Resistencia de manera masiva.

El trabajo de Alba Martínez también se adentra en las "zonas grises" de la Francia ocupada. En su caso, a partir de la reconstrucción de la experiencia de una joven refugiada que convivió estrechamente con los ocupantes. Una fuente excepcional como el diario de Conchita Martínez permite, sin embargo, profundizar en los parámetros de una vida "corriente" y afirmar que, aunque marginadas de los principales relatos sobre el exilio de 1939, las mujeres no fueron meras "acompañantes", sino que desplegaron una importante capacidad de agencia y adaptación a sus lugares de destino. Este artículo

\footnotetext{
22 Susan Keith, "Collective Memory and the End of Occupation: Remembering (and Forgetting) the Liberation of Paris in Images", Visual Communication Quarterly, 17:3 (2010), 134-146. La última publicación que elude calificar la "Liberación" como una ocupación es Gerd-Rainer Horn, The Moment of Liberation in Western Europe. Power Struggles and Rebellions (Oxford, Oxford University Press, 2020), 17-68.
} 
se pregunta por los perfiles cotidianos de la vida bajo la ocupación a partir de cuatro dimensiones, tanto físicas como emocionales: el lugar de trabajo, el hogar y la comunidad, los espacios de ocio y sociabilidad en Burdeos y la ciudad después de la "Liberación". En la mejor tradición de la microhistoria, esta contribución supone una reivindicación de lo "excepcional - normal" como principio analítico, que en el caso específico de la ocupación francesa supone adentrarse en los espacios de cohabitación con los invasores y la consiguiente difuminación de las categorías de "amigo" y "enemigo" en la vida cotidiana. También en la compleja, y a veces contradictoria, construcción de la subjetividad a partir de un contexto emocional particular como el bélico, en el que espacios como el cine, el tranvía o la propia calle adquieren una relevancia especial.

La ocupación de Francia en 1940 es interesante porque como sostuvo Bob Moore, a diferencia del frente oriental, el régimen de ocupación nazi en el Oeste tenía como objetivo mantener un alto grado de normalidad respecto al contexto de preguerra. Así, el caso francés tiene bastantes similitudes con el holandés, aunque quizá la principal diferencia radique en la relación que mantuvieron las autoridades locales con las ocupantes. Después del impacto causado por el bombardeo de Rotterdam, la reina Guillermina marchó al exilio en Londres junto al gobierno. Desde allí llamó a la colaboración de todos los funcionarios civiles con los alemanes, lo que dejó a las autoridades, especialmente las locales, en una posición ambivalente muy complicada de gestionar: por un lado, eran las más cercanas a las preocupaciones y necesidades de la población; por otro, fueron explotadas como instrumentos del gobierno autoritario, agentes de vigilancia y persecución racial $^{23}$. La historiografía holandesa ha alcanzado un alto grado de consenso a la hora de identificar la crisis en la relación de "viejas" y "nuevas" autoridades como el principal motivo de la radicalización del régimen de ocupación nazi y la singularidad que adquirió la persecución judía en esta latitud. No obstante, el contexto de la guerra también influyó notablemente en la gestión del orden,

\footnotetext{
${ }^{23}$ Esta situación es lo que todavía se conoce comúnmente como el "mal menor". Un balance completo en Peter Romijn, "«The Lesser Evil». The case of the Dutch local authorities and the Holocaust", en The Persecution of the Jews in the Netherlands, 1940-1945, Peter Romijn et al. (Ámsterdam, NIOD : Vossiuspers UvA, 2012), 13-26. El desarrollo anterior en Bob Moore, "Nazi Masters and Acomodating Dutch Bureaucrats: Working Towards the Führer", en Working Towards the Führer. Essays in Honour of Sir Ian Kershaw, Ed. Anthony McElligott y Tim Kirk (Manchester : New York, Manchester University Press, 2003), 186-204.
} 
como ocurrió igualmente en el caso francés. Según Louis De Jong, cuando esa crisis se mostró como irreversible de 1941 en adelante la administración foránea se basó en tres armas principales: el miedo, la división y la decepción ${ }^{24}$. Al igual que en la "Liberación” de Francia, la presencia nazi en Países Bajos terminó nuevamente con el Ejército asumiendo el papel principal en la gestión de un orden que, esta vez, residió en la colaboración cívico-militar ${ }^{25}$.

La crisis del régimen de ocupación nazi en Países Bajos aparece retratada en el artículo de Ingrid de Zwarte a partir de la hambruna de 1944-1945. La apuesta por la complejidad en el estudio de las ocupaciones rechaza de nuevo cualquier tipo de explicación monocausal y pasa por analizar las múltiples dimensiones del "Invierno del Hambre" más allá de las autoridades alemanas de ocupación. A través del original análisis de fuentes de origen diverso (alemanas, holandesas y del Alto Mando aliado), este artículo plantea que las causas que originaron la hambruna y la agravaron se encuentran en el progresivo deterioro de las condiciones de abastecimiento y transporte durante los compases finales de la II Guerra Mundial. En este contexto, las "nuevas" autoridades alemanas buscaron la colaboración con los propios funcionarios holandeses para evitar que las condiciones de vida empeorasen aún más y la situación no pusiera en riesgo del orden de ocupación, que dependía de un equilibrio inestable en términos locales y también en relación a la propia evolución del conflicto. Así, desde el punto de vista del Alto Mando aliado y del gobierno holandés en el exilio, el hambre se convirtió en un arma para desestabilizar la ocupación alemana, pero la huelga ferroviaria convocada en septiembre de 1944 afectó profundamente a la población y se unió a otras medidas tomadas por los alemanes para mantener el esfuerzo bélico de retaguardia en el marco de una "guerra total".

\footnotetext{
${ }^{24}$ Louis De Jong, The Netherlands and Nazi Germany (Cambridge : London, Harvard University Press, 1990); Dan Michman, "The Uniqueness of the Joodse Raad in the Western European Context", en Dutch Jewish History, Vol. III, ed. Jozeph Michman (Jerusalem, 1993), 371-380; Peter Romijn, "Managing the integration of the occupied Dutch territories in Hitler's empire", en Entrepeneurship in schwrierigen Zeiten. Unternehmertuum, Karrieren und Umbrüche während der ersten Hälfte des 20. Jahrhunderts, Hg. Peter Eigner; Herbert Matis y Andreas Resch (Wien, Liet Verlag, 2013), 309-330.

${ }^{25}$ Peter Romijn, “«Liberators and Patriots». Military Interim Rule and the Politics of Transition in Netherlands, 1944-1945", en Seeking Peace in the Wake of War. Europe, 1943-1947, eds. StephanLudwig Hoffmann et al. (Amsterdam, Amsterdam University Press, 2015), 117-144.
} 
La ocupación de los Países Bajos es un observatorio privilegiado para analizar la relación entre las autoridades locales y las foráneas, algo que forma parte esencial del trabajo que Peter Romijn presenta en este dossier. Hoy en día, 75 años después de que la ocupación nazi terminara, todavía es común escuchar la expresión "sentirse como un alcalde en tiempo de guerra". Las tensiones, los desafíos y las presiones a las que se enfrentaron los alcaldes holandeses durante la II Guerra Mundial se deben a una combinación de factores que explican el modo en que la propia ocupación del país tuvo lugar: la apuesta del gobierno en el exilio de colaborar con las autoridades alemanas, la administración civil de ocupación (contraria a la Convención de La Haya de 1907), el proceso de nazificación de los funcionarios holandeses y su papel en la persecución política y la deportación o la propia influencia del contexto geopolítico en la gestión de la administración y el control de la población. La aportación de Peter Romijn también es clave para plantear otra cuestión relevante: ¿desde qué parámetros se reconstruye una sociedad atravesada por la experiencia traumática de la ocupación?

Por último, mi propia contribución funciona como hilo conductor de las diversas aportaciones de este dossier, a modo de coda final. A partir de una mirada comparada de las ocupaciones de Madrid, Ámsterdam y París, mi artículo ofrece una reflexión sobre este fenómeno transnacional desde los parámetros de la historia urbana. De este modo, me pregunto por la influencia de fenómenos típicamente asociados a la ciudad como el anonimato, la movilidad, la delicada distribución de recursos o su propia escala física en el mantenimiento del orden de los regímenes de ocupación iniciados en 1939, 1940, 1944 y 1945 en España, Países Bajos y Francia. El texto se estructura a partir de tres estudios de caso que responden a tres cuestiones específicas. En primer lugar, cómo se expresó el tipo de administración, civil o militar, en cada una de las ciudades, con el análisis concreto de las causas y consecuencias urbanas de la "huelga de febrero" en Ámsterdam. En segundo lugar, de qué maneras se desarrolló la colaboración de la población con las autoridades de ocupación, una realidad que analizo de manera específica a través del caso de los "falsos policías" en París. En tercer y último lugar, la ocupación de Madrid a finales de la Guerra Civil alude a la relevancia de preguntarse tanto por las rupturas como por las continuidades que atraviesan la construcción de cualquier orden posconflicto. 
A mediados de la década de 1960, la revuelta Provo ya se había extendido desde la plaza Spui, en el centro de Ámsterdam, a todos los rincones de la ciudad, sacudiendo algunos de los consensos sociales y culturales más arraigados en la sociedad holandesa de posguerra. Combinando la no-violencia con otros nuevos repertorios de acción, como el happening, el arte urbano o el humor absurdo, los Provo acabaron por ser identificados por la policía como una de las principales alteraciones del orden durante aquellos años. Éste fue el contexto en que Frans Perrick, uno de los jefes de Policía desde 1945, reclamó reformas que transitaran hacia un nuevo sistema de orden público en el conjunto del Estado. Sabía de lo que hablaba. En 1968, volvió la vista hacia los efectos que la ocupación nazi había tenido en la policía de la ciudad, y sin embargo sus recuerdos se enfrentaron al silencio de una sociedad que había decidido olvidar aquel episodio traumático ${ }^{26}$. Había pasado justo una década desde que, en Francia, Maurice Papon fuera nombrado prefecto de la policía de París. Desde su puesto, fue el encargado de reprimir a sangre y fuego la manifestación que el argelino Frente de Liberación Nacional convocó el 17 de octubre de 1961 en la capital. Dependiendo de las fuentes, el balance de aquella jornada oscila entre 38 y más de 200 muertos. Años después de aquel acontecimiento, la sociedad francesa conoció su responsabilidad en la deportación de judíos desde su cargo en la prefectura de la Gironda, bajo el régimen de Vichy ${ }^{27}$. En 2014, el Consejo Superior de Investigaciones Científicas celebró su 75 aniversario reivindicándose como heredero directo de la Junta para la Ampliación de Estudios, obviando que la fundación de uno y la desaparición de la otra representaban dos caras de la misma moneda: la ocupación de las instituciones académicas al final de la Guerra Civil española ${ }^{28}$.

“¿Cuándo termina realmente una ocupación?” Esta cuestión, presente en un intercambio por correo electrónico con uno de los participantes en este dossier, sobrevuela de manera general todas las contribuciones. Es probable que sepamos

\footnotetext{
${ }^{26}$ Cyrille Fijnaut et al., "The Impact of the Occupation on the Dutch Police", en The Impact of World War II on Policing in North-West Europe, ed. Cyrille Fijnaut (Leuven, Leuven University Press, 2004), 91-132.

27 Sobre las diferentes aristas del "affaire Papon", puede verse Richard Golsan (Ed.), The Papon affair: memory and justice on trial (Routledge, Londres, 2000). Agradezco a José Luis Ledesma esta referencia.

${ }^{28}$ Alba Fernández Gallego, “«Donde habita el olvido». La apropiación de la Colina de los Chopos en el nuevo Madrid científico (1939-1948)", en Madrid, una ciudad en guerra (1936-1948), coords. Daniel Oviedo Silva y Alejandro Pérez-Olivares (Madrid, Los Libros de la Catarata, 2016), 214-261.
} 
identificar más fácilmente cuándo comienza una ocupación. Algunas de sus estampas aparecen muy marcadas en nuestro imaginario colectivo: la elevación de una barrera, en el caso de invadir un país, o la entrada en una ciudad y el desfile de un ejército por sus principales avenidas, por ejemplo. Cada uno de los textos que ahora se presentan han abordado tanto la pregunta directa como la aparente certeza de manera amplia, "liberando" el estudio de las ocupaciones del peso de las imágenes y de las certidumbres, y situándolo en medio de todas las transformaciones y todas las permanencias que componen su historicidad. Tener la oportunidad de haber coordinado este dossier supone la prolongación de algunas reflexiones que empecé a desarrollar durante mi estancia de investigación en el NIOD Institute for War, Holocaust and Genocide Studies de Ámsterdam, bajo la supervisión del profesor Peter Romijn. Es extraño poner por escrito, a finales de 2020, ideas que empezaron a surgir en el otoño de 2016, trabajar sobre notas y perspectivas que me acompañaron en mis paseos a lo largo del Herengracht hace cuatro años. Sin embargo, mis intereses apenas podrían comprenderse ahora sin todos los trabajos que han enriquecido cualquier interpretación individual y que han transformado gran parte de esas intuiciones iniciales.

En este sentido, mi gratitud es infinita hacia las autoras y los autores que componen este trabajo coral, una nueva muestra de que ninguna idea es posible de manera aislada, y de que las mejores respuestas son siempre colectivas. Esta introducción es el lugar más apropiado para reconocer su esfuerzo en un tiempo de excepción, sin duda, en el que las sucesivas etapas de redacción, entrega y revisión de los textos han vencido confinamientos y reconfinamientos, desescaladas y toques de queda. Mi agradecimiento también se dirige a los editores de Hispania Nova, a su consejo de redacción y su secretaría técnica, por su confianza inicial y su paciencia constante. También por su esfuerzo. Pionera como revista online de historia contemporánea en castellano, el compromiso de Hispania Nova con la difusión de los debates académicos más recientes y relevantes adquiere esta vez la forma de un dossier íntegramente en nuestro idioma. Así, los estudios de Jean Marc Berlière, Ingrid de Zwarte y Peter Romijn que aparecen en este número se presentan traducidos por vez primera tanto a la comunidad académica como al gran público. Estoy convencido de que muy pronto formarán parte de los debates de nuestra historiografía y se convertirán en referencias ineludibles para comprender el pasado de manera más satisfactoria. Ya lo 
son para ampliar las experiencias, los rostros y los espacios que definen cualquier tiempo marcado por la ocupación. Espero que estas páginas y las siguientes nos ayuden, dentro y fuera de la Universidad, a "estirar el cuello" y descubrir nuevos sentidos en la realidad que nos rodea, como recordaba la historiadora Arlette Farge desde la sala de un archivo.

\section{BIBLIOGRAFÍA.}

Agamben, Giorgio, "Reflexiones sobre la peste", en Giorgio Agamben et al., Sopa de Wuhan, Editorial ASPO, 2020, 135-137

Agamben, Giorgio, Estado de excepción. Homo Sacer II, 1(Madrid, Pre-Textos, 2010).

Alonso Ibarra, Miguel, "Combatir, ocupar, fusilar. La evolución de la violencia bélica de los sublevados en la guerra civil española (1936-1939)", en Alegre Lorenz, David; Alonso Ibarra, Miguel y Rodrigo, Javier (coords.), Europa desgarrada: guerra, ocupación y violencia (1900-1950), Zaragoza, Prensas Universitarias de Zaragoza, 2018.

Amnistía Internacional, Violaciones de derechos humanos durante el estado de alarma (Madrid, 2020), https://www.es.amnesty.org/fileadmin/user_upload/Violaciones_ddhh_estado_d e_alarma_junio_2020.pdf

Boldorf, Marcel y Okazaki, Tetsuki Okazaki (eds.), Economies Under Occupation. The Hegemony of Nazi Germany and Imperial Japan in World War II (London: New York, Routledge, 2015)

Bowman, Steve; Kapp, Lawrence y Belasco, Amy, Hurricane Katrina: DOD Disaster Response, Washington, The Library of Congress, 2005, https://fas.org/sgp/crs/natsec/RL33095.pdf

Broche, François, Dictionnaire de la Collaboration. Collaborations, compromissions, contradictions (Paris, Belin, 2014).

Burrin, Philippe, "Writing the History of Military Occupations", en Fishman, Sarah (ed.), France at War: Vichy and the Historians, Oxford, Berg, 2000, 77-90.

Collins, Joseph J., Choosing War. The Decision to Invade Iraq and its Aftermath, Washington DC, National Defense University Press, 2008.

Cruz, Rafael, En el nombre del pueblo. República, rebelión y guerra en la España de 1936 (Madrid, Siglo XXI, 2006).

De Jong, Louis, The Netherlands and Nazi Germany, Cambridge : London, Harvard University Press, 1990. 
De Schaepdrijver, Sophie, "Military occupations, 1914-1945", en Chickering, Roger; Showalter, Dennis y Van De Ven, Hans (eds.), The Cambridge History of War, Vol. IV. War and the Modern World, Cambridge, Cambridge University Press, 2012, 236-256.

--, "Populations under occupation", en Winter, Jay (ed.), The Cambridge History of the First World War. Vol. III: Civil Society, Cambridge, Cambridge University Press, 2014, 242-256.

Debrash, Odile, L'occupation militaire, Paris, Librairie générale de droit et de jurisprudence, 1962.

Egoz, Shelley Egoz y Williams, Tim, “Coexistent Landscapes: Military Integration and Civilian Fragmentation”, en Chris Pearson, Peter Coates y Tim Cole (Ed.), Militarized Landscapes. From Gettysburg to Salisbury Plan, London : New York, Continuum Books, 2010.

Farge, Arlette, La atracción del archivo, Valencia, Edicions Alfons el Magnànim, 1991.

Fernández Gallego, Alba, “«Donde habita el olvido». La apropiación de la Colina de los Chopos en el nuevo Madrid científico (1939-1948)", en Daniel Oviedo Silva y Alejandro Pérez-Olivares (coords.), Madrid, una ciudad en guerra (1936-1948), Madrid, Los Libros de la Catarata, 2016, 214-261.

Fijnaut, Cyrille et al., "The Impact of the Occupation on the Dutch Police", en Fijnaut, Cyrille (ed.), The Impact of World War II on Policing in North-West Europe, Leuven, Leuven University Press, 2004, 91-132.

Fraenkel, Ernst, Military Occupation and the Rule of Law, New York, Oxford University Press, 1944.

Franch-Pardo, Ivan et al., "Spatial Analysis and GIS in the study of COVID-19. A study", Science of the Total Environment, 739 (2020), doi: https://doi.org/10.1016/j.scitotenv.2020.140033.

Gildea, Robert, Marianne in Chains. Daily Life in the Heart of France During the German Ocupation, London, MacMilan, 2002.

Godicheau, François, "Guerra civil, guerra incivil: la pacificación por el nombre", en Godicheau, François y Aróstegui, Julio (Coord.), Guerra civil: mito y memoria, Madrid, Marcial Pons, 2006, 137-166.

--, "La Guerra Civil, figura del desorden público. El concepto de guerra civil y la definición del orden político", en Canal, Jordi y González Calleja, Eduardo (eds.), Guerras civiles. Una clave para entender la Europa de los siglos XIX y $X X$, eds. (Madrid, Casa de Velázquez, 2012), 75-88.

Golsan, Richard (Ed.), The Papon affair: memory and justice on trial, Routledge, Londres, 2000.

Gómez Bravo, Gutmaro, Geografía humana de la represión franquista. Del golpe a la guerra de ocupación, 1936-1941, Madrid, Cátedra, 2017

Gómez Bravo, Gutmaro y Marco, Jorge, La obra del miedo. Violencia y sociedad en la España franquista, 1936-1950, Barcelona, Península, 2011. 
Hinnebusch, Raymond, "The US Invasion of Iraq: Explanations and Implications", Critique: Critical Middle Eastern Studies (2007), 209-228.

Horn, Gerd-Rainer, The Moment of Liberation in Western Europe. Power Struggles and Rebellions, Oxford, Oxford University Press, 2020.

Keith, Susan, "Collective Memory and the End of Occupation: Remembering (and Forgetting) the Liberation of Paris in Images", Visual Communication Quarterly, 17:3 (2010), 134-146.

Kitson, Simon, "The Police in the Liberation of Paris", en Kedward, H. R. y Wood, Nancy (ed.), The Liberation of France. Image and Event, Oxford : Herndon, Berg, 1995, 43-56.

Lammers, Cor, "Levels of Collaboration: A Comparative Study of German Occcupation Regimes during the Second World War", The Netherlands Journal of Social Sciences, 31 (1995), 3-31.

Laub, Thomas J., After the Fall. German Policy in Occupied France, 1940-1944, New York, Oxford University Press, 2010.

Marco, Jorge, "Rethinking the Postwar Period in Spain: Violence and Irregular Civil War, 1939-52”, Journal of Contemporary History, 55/3 (2019), 492-513

Mazower, Mark, El imperio de Hitler, Barcelona, Crítica, 2008.

Michman, Dan, "The Uniqueness of the Joodse Raad in the Western European Context", en Michman, Jozeph (ed.), Dutch Jewish History, Vol. III, Jerusalem, 1993, 371-380.

Moore, Bob, "Nazi Masters and Acomodating Dutch Bureaucrats: Working Towards the Führer", en McElligott, Anthony y Kirk, Tim (Ed.), Working Towards the Führer. Essays in Honour of Sir Ian Kershaw, Manchester : New York, Manchester University Press, 2003, 186-204.

Mouralis, Guillaume, "Le procès Papon. Justice et temporalité”, Terrain, 38 (2002), 5568, doi : https://doi.org/10.4000/terrain.9953.

Mouré, Kenneth, "The Faux Policier in Occupied Paris", Journal of Contemporary History, 45/I (2010), 95-112.

Oscar, Christopher, Post Katrina Redefining the Military Role in Homeland Security, US Army War College, Carlisle, 2006.

Paisaje "Lansversal, ciudad del Coronavirus", https://paisajetransversal.org/2020/07/la-ciudad-del-coronavirus/

Pérez-Olivares, Alejandro, Madrid cautivo. Ocupación y control de una ciudad, 19361948, Valencia, Publicacions de la Universitat de València, 2020.

Ramos Tolosa, Jorge, "Propuestas para decolonizar Palestina-Israel", en Maria Paula Meneses y Karina Bidaseca (eds.), Epistemologías del Sur, Buenos Aires : Coimbra, CLACSO, 2018.

Raymond, Robin, Des occupations militaires en dehors des occupations de guerre, Paris, Larose \& Tenin, 1913. 
Rodrigo, Javier, Cruzada, paz, memoria. La Guerra Civil en sus relatos, Granada, Comares, 2013.

Rodrigo, Javier y Alegre, David, Comunidades rotas. Una historia global de las guerras civiles, 1917-2017, Barcelona, Galaxia Gutenberg, 2019.

Romijn, Peter y Tames, Ismee, "Transnational Identities of Dutch-Nazi Collaborators and their Struggle for Integration into the National Community", Journal of Modern European History, 16/2 (2018), 247-268.

Romijn, Peter, "«Liberators and Patriots». Military Interim Rule and the Politics of Transition in Netherlands, 1944-1945", en Hoffmann, Stephan-Ludwig et al. (eds.), Seeking Peace in the Wake of War. Europe, 1943-1947, Amsterdam, Amsterdam University Press, 2015, 117-144.

--, "«The Lesser Evil». The case of the Dutch local authorities and the Holocaust", en Romijn, Peter et al., The Persecution of the Jews in the Netherlands, 19401945, Ámsterdam, NIOD : Vossiuspers UvA, 2012, 13-26.

--, "Managing the integration of the occupied Dutch territories in Hitler's empire", en Eigner, Peter; Matis, Herbert y Resch, Andreas (Hg.), RescEntrepeneurship in schwrierigen Zeiten. Unternehmertuum, Karrieren und Umbrüche während der ersten Hälfte des 20. Jahrhunderts, Wien, Liet Verlag, 2013, 309-330.

Sánchez León, Pablo, "Erradicar la ciudadanía: 1936 más allá de una guerra civill”, en Sánchez León, Pablo e Izquierdo Martín, Jesús, La guerra que nos han contado y la que no. Memoria e historia de 1936 para el siglo XXI, Madrid, Postmetrópolis, 2017, 305-380.

Scala, Antonio et al., "Time, space and social interactions: exit mechanisms for the Covid-19 epidemics", Scientific Reports, $10 \quad$ (2020), doi: https://doi.org/10.1038/s41598-020-70631-9

Showalter, Dennis, "Military occupations, 1945-1955", en Chickering, Roger; Showalter, Dennis y Van De Ven, Hans (eds.), The Cambridge History of War, Vol. IV. War and the Modern World, Cambridge, Cambridge University Press, 2012, 428-451.

Thénault, Sylvie, 'L'état d'urgence (1955-2005). De l'Algérie coloniale à la France contemporaine: destin d'une loi”, Le Mouvement Social, 218/1 (2007), 63-78

Traverso, Enzo, A sangre y fuego. De la guerra civil europea, 1914-1945, Valencia, Publicacions de la Universitat de València, 2009.

Vinen, Richard, The Unfree French. Life Under Occupation, London, Penguin, 2007. 